\title{
Thermodynamic analysis of vapour compression refrigeration system using alternative refrigerants
}

\author{
Monika Singh $^{1}$, Prashant Somvanshi ${ }^{2}$ \\ Asst professor, mechanical engineering, Ajmer institute of technology, (Rajasthan $)^{1 \& 2}$
}

\begin{abstract}
This research is aimed to define, (1) the effects of refrigerant to the environment, (2) derive the eco friendly refrigerants by using software REFPROP, (3) describe the properties of refrigerant by mixing them in appropriate percentage, (4) reducing GWP and ODP by providing suitable substitute of CFC's and HCFC's refrigerants, (5) reducing the effect of refrigerant to the ODP.

This analysis is executed through data collection, calculation for COP and analysis of refrigerant effect and compressor work using REFPROP, environmental impact of refrigerant and provides the literature review of research for eco-friendly refrigerant.

Keywords: Software for calculating Refrigeration properties of composite refrigerants (REFROP), Global warming Potential (GWP), Ozone Depletion Potential (ODP), chlorofluorocarbon (CFC). Hydrochlorofluorocarbons (HCFC)
\end{abstract}

\section{Introduction}

Now a day CFC and HCFC composites refrigerant are being gradually eliminated because of their implication in destruction of ozone layer and in the increase of greenhouse effect. . To promote and manage a gradual process of elimination and substitution for the CFCs, some countries were congregated in 1985 in the Convention of Vienna and started an agreement that was firmed up in 1987 in Canada, the Protocol of Montreal. This protocol determined the elimination of the CFCs until 1996 and the HCFCs until 2030 in the developed countries and a lack of 10 years in the developing countries (GTO-Interminesterial Ozone Working Group, 1994) .Besides chlorine in their constitution, these composites are characterized by the great stability, what makes them persist in the terrestrial atmosphere for many years. From the commercial point of view, the CFCs R-11 and R-12 and the HCFCs R-22 and R-502 substitution became the most important. Table 1 shows the environmental impacts of refrigerants R12 and R22 through the Global Warming Potential (GWP) and the Ozone Depleting Potential (ODP).These indexes characterize the action level of these chemical composites on the ozone layer and the greenhouse effect, besides the atmosphere lifetime.

\begin{tabular}{|l|l|l|l|l|}
\hline Refrigerants & Chemical formula & $\begin{array}{l}\text { Time in the } \\
\text { atmosphere }\end{array}$ & $\begin{array}{l}\text { Global warming } \\
\text { potential(GWP) }\end{array}$ & $\begin{array}{l}\text { Ozone depletion } \\
\text { potential (ODP) }\end{array}$ \\
\hline R12 & $\mathrm{CCl}_{2} \mathrm{~F}_{2}$ & 100 & 8500 & 1.0 \\
\hline $\mathbf{R 2 2}$ & $\mathrm{CHClF}_{2}$ & 12 & 1700 & 0.05 \\
\hline
\end{tabular}

R22 is widely used, both in terms of refrigeration capacity and for commercial application. It is the refrigerant adopted by the great majority of the refrigeration equipment in operation, being used in the most different economic sectors: residential, commercial, industrial and transport. The hydro fluorocarbon (HFC) R134a is being used to substitute for this composite. However, this transition does not happen easily, mainly in function of lubricant compatibility problems. Another refrigerant that has attracted interest to substitute for R22 is R410a, a near-azeotrope mixture of R32 and R125 of 50-50\% per mass. At the same time, different research lines are being followed, with a renewed interest in natural refrigerants, as the hydrocarbons propane (R290), isobutene (R600a) and their mixtures, mainly for its lower values of GWP and null ODP that can be observed in the Tab. 2, together with the properties of R134a and R410a.

\begin{tabular}{|l|l|l|l|l|}
\hline Refrigerants & Chemical formula & Time in atmosphere & $\begin{array}{l}\text { Global } \\
\text { potential(GWP) }\end{array}$ & $\begin{array}{c}\text { Ozone } \\
\text { potential(ODP) }\end{array}$ \\
\hline R600a & $\mathrm{C}_{4} \mathrm{H}_{10}$ & $<1$ & $<20$ & 0 \\
\hline R290 & $\mathrm{C}_{3} \mathrm{H}_{8}$ & $<1$ & $<20$ & 0 \\
\hline R134a & $\mathrm{CH}_{2} \mathrm{FCF}_{3}$ & 14 & 1600 & 0 \\
\hline R410a & -------- & ------ & 2340 & 0 \\
\hline
\end{tabular}

Despite the values indicated in Tab. 2, the effect of a refrigerant in the global heating must be analyzed in a broad way: on the one hand there is the direct contribution due to the refrigerant leakages and on the other hand there is the indirect contribution due to the energy consumption to operate the system. The Total Equivalent 
Warming Impact(TEWI) term combines both direct and indirect equivalent $\mathrm{CO} 2$ contributions and has been used in the environmental effect evaluation of refrigerant systems (Sand et all, 1999). The hydrocarbons have low or no direct effect in the global heating. However when the system is operating they can have a comparable indirect heating to R134a. Higher energy consumption is associated to more fuel burning and, therefore, more $\mathrm{CO} 2$ release. This effect can be reduced with more efficient equipment.

Thus, the evaluation of natural refrigerants must not only include environmental aspects, but the performance criteria, energy consumption besides the security and physical-chemical stability. Associated to a good performance, there is the matter of the conversion of the existing equipment that can include compressors design, heat exchangers, control systems, lubricant and materials compatibility.

Therefore our work concentrates on the study of environmental aspects as well as energy consumption aspect of various refrigeration.Our work focused on the thermodynamic analysis of various refrigerants using vapour compression refrigeration cycle. Various refrigerants are now coming up as substitutes to replace R22 and R134a. R134a is immensely used in refrigeration industry these days. This HFC although is inflammable but contributes to global warming a lot. Besides this environmental issue, R134a is not compatible with mineral oil. Therefore in case of retrofitting oil change is going to be a major issue. In this present work a theoretical analysis has been done on various refrigerants which include R134a, R22, R290, R410a, R407c, and MO9 (mixture of R134a/R290/R600a). Coefficient of performance of these refrigerants has been compared. Thermodynamic properties of these refrigerants are taken from REFPROP software(NIST).In present study M09 found out to be a suitable substitute for R134a for new system as well as for retrofitting.

\section{WHAT IS A REFRIGERANT?}

Webster's dictionary defines refrigerants as "a substance used in a refrigerating cycle ordirectly such as ice for cooling". Water is the refrigerant used in absorption chillers. Carbon Dioxide (CO2) and Ammonia (NH3) are known as "natural" refrigerants. Flammable substances such as propane and isobutene are also used as refrigerants. To this group, CFCs, HCFCs and HFCs can be added.

HCFC-22 is the most widely used refrigerant in the world. HCFC-123 is the interim replacement for CFC11. They are used in vapor compression processes will all types of compressors. HCFC22 is efficient and classified as A1 (lower toxicity - no flame propagation). HCFC-123 is efficient and classified as B1 (higher toxicity -no flame propagation).

Like CFCs, these refrigerants are being phased out as required by the Montreal Protocol. Production has been capped and soon will be ratcheted down in developed countries. Developing countries also have a phase-out schedule but on an extended timeline.

HFCs have zero ODP. HFC-134a is the replacement for CFC-12 and R-500.

\section{Basic Definitions Related To Rfrigerant}

There are many properties of refrigerants which are very much required to be fulfilling their criteria of range. For examples efficiency should be maximized toxicity and flammability should be minimized,Material compatibility, boiling temperature, freezing point, critical point, ODP and GWP which are discussed in this paper.

Ozone Depletion Potential: ODP is an index of a substance's ability to destroy atmospheric ozone. Table 1 list the ODP for many common refrigerants. Note that R-11 and R-12 have the highest potential (1.0). while the ODP of the refrigerant does not affect its performance as a refrigerant, it is a key parameter. All new refrigerant/product development must bebased on refrigerant with no ODP.

Global Warming Potential: GWP is an index of a substance's ability to be green house gas. GWP is related to the warming effect of a similar mass of a carbon dioxide for a 100-year time frame. Carbon dioxide is used as reference gas as it has the greatest net impact on global warming. Halocarbon refrigerants typically have higher GWPs than carbon dioxide but are in much smaller quantities.

GWP Consideration: R-134a is an HFC refrigerant. HFCs are one of six chemicals in a "basket" that are specifically signaled out by the Kyoto Protocol on global warming as a greenhouse gas. While this means countries attempting to meet the requirements of the Kyoto Protocol must be careful how they use HFCs, it does not mean there is a phase out. There is no reason for HFCs not to be considered a viable solution as a refrigerant now or in the future. A better measure of the warming impact of refrigerants is TEWI.

\section{Refrigerant On Which I Have Worked}

PROPANE AND ISOBUTANE: Propane and isobutane have low toxicity, good performance, no ODP and low GWP. However, they are flammable. Northern Europe has accepted them in refrigerators. Coca-Cola has announced it will move away from HFCs and has considered flammable refrigerants. In the United States, there are strong concerns about safety with respect to flammability. There have been studies performed evaluating the efficiency of flammable refrigerants where brine is cooled and then pumped to where cooling is required (as 
opposed to direct DX cooling). These reports show propane and isobutane as less efficient than traditional direct cooling R-22 systems. However this should be understood in light of the use of brine. There are only a handful of large capacity installations in the world.

R-134: R-134a is classified as an A1 (Lower toxicity - no flame propagation) refrigerant by ASHRAE, it has no ODP but the GWP is 1300. It has very good performance, heat transfer properties and is a good candidate for screw and centrifugal compressor applications. Its acceptance in the automotive industry makes it very abundant.

R-134a appears to be caught between the Montreal and Kyoto Protocols. The Montreal Protocol is systematically removing from service common refrigerant with ODP $>0$. By default, the protocol is driving the market to refrigerants with ODP=0 such as R-134a. On the other hand, the Kyoto protocol has put HFCs in the basket of targeted gases.

$\mathrm{R}-134 \mathrm{a}$ is one of the best solutions available. It has 0 ODP and therefore has no phaseout date from the Montreal Protocol. Although HFCs are in the Kyoto Basket, only its emissions are regulated. There is no phase out date for HFCs. While CFCs and HCFCs are major contributors to ozone depletion (28\% of anthropogenic ozone depletion), HFCs, CFCs and HCFCs direct effect is only a minor player in climate change (4\% of anthropogenic global warming).

R-407C: R-407C is a zeotropic blend of HFC-32, HFC-125 and HFC-134a. Its properties have been "tuned" to be very close to R-22 but it has a glide of over $8^{\circ} \mathrm{F}$. R-407C can be "dropped" into an existing R-22 refrigeration system and work although often with some performance loss. In many applications, the performance can be improved with minor changes to the refrigeration system sub-components (for example adding condenser surface area).

The high glide limits its applications to only to DX units such as rooftop units and some chillers where glide is less of an issue. R-407C is often considered a "replacement drop in" refrigerant to be used in upgrading existing systems to an HFC refrigerant. There is limited new generation product development based on R-407C.

R-410A: R-410A is a zeotropic blend of HFC-32 and HFC-125. It has a minimal glide (less that $1{ }^{\circ} \mathrm{F}$ ) and a very low volume flow rate $(1.5 \mathrm{Cfm} /$ tonne). It also operates at higher pressures than R-22 (450 psi). It cannot be dropped into an existing R-22 system but instead must be used in a new generation design.

One issue with R-410A is the low critical point and applications in high ambient. This significantly reduces the performance of air cooled equipment in hot locations. Water-cooled equipment is generally unaffected because of the lower condensing temperatures.

Chloridifluromethane (R-22): R22 is a single hydro chlorofluorocarbon or HCFC compound. Low chlorine content and ozone depletion potential, $\mathrm{ODP}=0.05$. Modest global warming potential GWP $=1700$.

R22 can still be used in small heat pump systems, but new systems can not be manufactured for use in the EU after 2003. From 2010 only recycled or saved stocks of R22 can be used. It will no longer be manufactured. Little odor, colorless as gas or liquid, non toxic, nonirritating, non flammable, non corrosive, stable. Itcan be used inpackaged air-conditioning units where size of equipment and economy are important, Air Conditioning, Low and Medium Temperature Refrigeration.

\section{REFPROP (NIST)}

REFPROP is an acronym for Reference fluid Properties. This program, developed by the National Institute of Standards and Technology (NIST), calculates the thermodynamic and transport properties of industrially important fluids and their mixtures. These properties can be displayed in tables and plots through the graphical user interface; they are also accessible through spreadsheets or user-written applications accessing the REFPROP DLL or the FORTRAN property subroutines.

REFPROP is based on the most accurate pure fluid and mixture models currently available. It implements three models for the thermodynamic properties of pure fluids: equations of state explicit in Helmholtz energy, the modified Benedict-Webb-Rubin equation of state, and an extended corresponding states (ECS) model.

Mixture calculations employ a model that applies mixing rules to the Helmholtz energy of the mixture components; it uses a departure function to account for the departure from ideal mixing. Viscosity and thermal conductivity are modelled with either fluid-specific correlations, an ECS method, or in some cases the friction theory method.

REFPROP has a list of pure fluids available and properties associated with it. We can also make a mixture of fluids from this list of pure fluids.

I used REFPROP version 9.0 for this analysis. 
ANALYSIS

COP of various refrigerants are calculated using following steps:

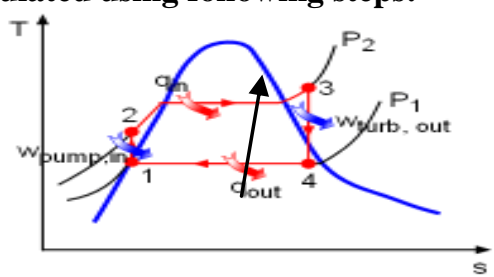

Values of enthalpy and entropy at saturation points i.e. pints 1, 4, 3', 2 are known from table obtained from REFPROP shown below. We need to find values of entropy and enthalpy at point 3. By using the formula-

$s_{3}=s_{3}{ }^{\prime}+\ln \left(T_{3} / T_{3}{ }^{\prime}\right)$

After finding out the value of $T_{3}$, value of enthalpy at point 3 is calculated using formula:

$\mathbf{h}_{3}=\mathbf{h}_{3},+\mathbf{c p}\left(\mathbf{T}_{3}-\mathbf{T}_{3},\right)$

Now,

COP $=\left(h_{1}-h_{4}\right) /\left(h 3-h_{4}\right)$

$\mathrm{h}_{\mathrm{v}}=$ enthalpy at vapour state

$\mathrm{h}_{1}=$ enthalpy at liquid state

$\mathrm{S}_{\mathrm{v}}=$ entropy at vapour state

$\mathrm{S}_{1}=$ entropy at liquid state

$\mathrm{C}_{\mathrm{ps}}=$ specific heat at liquid state

$\mathrm{C}_{\mathrm{pv}}=$ specific heat at vapour state

PROPANE(R290):

\begin{tabular}{|l|l|l|l|l|l|l|l|}
\hline $\mathbf{T}(\square \mathbf{C})$ & $\mathbf{P}$ & $\mathbf{h}_{\mathbf{v}}$ & $\mathbf{h}_{\mathbf{1}}$ & $\mathbf{S}_{\mathbf{1}}$ & $\mathbf{S}_{\mathbf{v}}$ & $\mathbf{C}_{\mathrm{ps}}$ & $\mathbf{C}_{\mathrm{pv}}$ \\
\hline $\mathbf{2 5 3}$ & & & & & & & \\
\hline $\mathbf{3 1 3}$ & 1.3640 & 551.96 & 151.01 & .81537 & 2.4001 & 2.3599 & 1.5792 \\
\hline
\end{tabular}

\begin{tabular}{|l|l|l|l|l|l|l|l|l|}
\hline $\mathbf{T}(\square \mathbf{C})$ & $\mathbf{P}$ & $\mathbf{h v}$ & $\mathbf{h}_{\mathbf{1}}$ & $\mathbf{S}_{\mathbf{1}}$ & $\mathbf{S}_{\mathbf{v}}$ & $\mathbf{C}_{\mathrm{ps}}$ & $\mathbf{C}_{\mathbf{p v}}$ \\
\hline $\mathbf{2 6 3}$ & .34357 & 563.48 & 174.98 & .90759 & 2.3848 & 2.4220 & 1.6536 \\
\hline $\mathbf{3 1 3}$ & 1.3647 & 614.09 & 306.71 & 1.3580 & 2.3400 & 2.9105 & 2.2602 \\
\hline $\mathbf{T}(\square \mathbf{C})$ & $\mathbf{P}$ & $\mathbf{h}_{\mathbf{v}}$ & $\mathbf{h}_{\mathbf{1}}$ & $\mathbf{S}_{\mathbf{1}}$ & $\mathbf{S}_{\mathbf{v}}$ & $\mathbf{C}_{\mathbf{p s}}$ & $\mathbf{C}_{\mathbf{p v}}$ \\
\hline $\mathbf{2 7 3}$ & .47229 & 574.70 & 199.62 & .99864 & 2.3725 & 2.4920 & 1.7374 \\
\hline $\mathbf{3 1 3}$ & 1.3647 & 614.09 & 306.71 & 1.3580 & 2.3400 & 2.9105 & 2.2602 \\
\hline
\end{tabular}

\begin{tabular}{|l|l|l|l|l|l|l|l|}
\hline $\mathbf{T}(\square \mathbf{C})$ & $\mathbf{P}$ & $\mathbf{h}_{\mathbf{v}}$ & $\mathbf{h}_{\mathbf{1}}$ & $\mathbf{S}_{\mathbf{1}}$ & $\mathbf{S}_{\mathbf{v}}$ & $\mathbf{C}_{\mathbf{p s}}$ & $\mathbf{C}_{\mathbf{p v}}$ \\
\hline $\mathbf{2 8 3}$ & .63390 & 585.51 & 225.01 & 1.0888 & 2.3627 & 2.5720 & 1.8337 \\
\hline $\mathbf{3 1 3}$ & 1.3647 & 614.09 & 306.71 & 1.3580 & 2.3400 & 2.9105 & 2.2602 \\
\hline
\end{tabular}

R22.

\begin{tabular}{|l|l|l|l|l|l|l|l|}
\hline $\mathbf{T}(\square \mathbf{C})$ & $\mathbf{P}$ & $\mathbf{h}_{\mathbf{v}}$ & $\mathbf{h}_{\mathbf{1}}$ & $\mathbf{S}_{\mathbf{1}}$ & $\mathbf{S}_{\mathbf{v}}$ & $\mathbf{C}_{\mathbf{p s}}$ & $\mathbf{C}_{\mathbf{p v}}$ \\
\hline $\mathbf{2 5 3}$ & 0.2439 & 397.0 & 176.87 & 0.91281 & 1.7829 & 1.1224 \\
\hline $\mathbf{3 1 3}$ & 1.528 & 416.22 & 249.45 & 1.1658 & 1.6987 & 1.3379 & 0.66454 \\
\hline
\end{tabular}

\begin{tabular}{|l|l|l|l|l|l|l|l|}
\hline $\mathbf{T}(\square \mathbf{C})$ & $\mathbf{P}$ & $\mathbf{h}_{\mathbf{v}}$ & $\mathbf{h}_{\mathbf{1}}$ & $\mathbf{S}_{\mathbf{1}}$ & $\mathbf{S}_{\mathbf{v}}$ & $\mathbf{C}_{\mathbf{p s}}$ & $\mathbf{C}_{\mathbf{p v}}$ \\
\hline $\mathbf{2 6 3}$ & 0.35291 & 401.14 & 188.23 & 0.95652 & 1.7660 & 1.1436 \\
\hline $\mathbf{3 1 3}$ & 1.528 & 416.22 & 249.45 & 1.1658 & 1.6987 & 1.3379 \\
\hline
\end{tabular}

\begin{tabular}{|l|l|l|l|l|l|l|l|}
\hline $\mathbf{T}(\square \mathbf{C})$ & $\mathbf{P}$ & $\mathbf{h}_{\mathbf{v}}$ & $\mathbf{h}_{\mathbf{l}}$ & $\mathbf{S}_{\mathbf{l}}$ & $\mathbf{S}_{\mathbf{v}}$ & $\mathbf{C}_{\mathbf{p s}}$ & $\mathbf{C}_{\mathbf{p v}}$ \\
\hline $\mathbf{2 7 3}$ & 0.49556 & 404.99 & 199.82 & 0.99936 & 1.7509 & 1.1688 & 0.73833 \\
\hline $\mathbf{3 1 3}$ & 1.528 & 416.22 & 249.45 & 1.1658 & 1.6987 & 1.3379 \\
\hline
\end{tabular}

\begin{tabular}{|l|l|l|l|l|l|l|l|}
\hline $\mathbf{T}(\square \mathbf{C})$ & $\mathbf{P}$ & $\mathbf{h}_{\mathrm{v}}$ & $\mathbf{h}_{\mathbf{l}}$ & $\mathbf{S}_{\mathbf{l}}$ & $\mathbf{S}_{\mathbf{v}}$ & $\mathbf{C}_{\mathrm{ps}}$ & $\mathbf{C}_{\mathbf{p v}}$ \\
\hline $\mathbf{2 8 3}$ & 0.67788 & 408.51 & 211.69 & 1.0415 & 1.737 & 1.1988 \\
\hline $\mathbf{3 1 3}$ & 1.528 & 416.22 & 249.45 & 1.1658 & 1.6987 & 1.3379 \\
\hline
\end{tabular}

R134a: 
Thermodynamic analysis of vapour compression refrigeration system using alternative refrigerants

\begin{tabular}{|c|c|c|c|c|c|c|c|}
\hline $\mathbf{T}(\square \mathrm{C})$ & $\mathbf{P}$ & $\mathbf{h}_{\mathrm{v}}$ & $\mathbf{h}_{\perp}$ & $S_{\perp}$ & $\mathbf{S}_{\mathrm{v}}$ & $\mathrm{C}_{\mathrm{pS}}$ & $\mathrm{C}_{\mathrm{DV}}$ \\
\hline $\left.283^{C}\right)$ & Ð.57315 & $\$ 24.33$ & 214.39 & $1 \mathbf{S} 0513$ & 1 \$.8168 & 1.40768 & 1.9466 \\
\hline $\mathbf{3 7 3}$ & P.:28188 & 4836.43 & $26 \hat{0}: 844$ & 1.289969 & $1.7473^{5}$ & 1.64927 & 1.38585 \\
\hline 313 & 1.0125 & 419.36 & 256.19 & 1.1898 & 1.7111 & 1.4975 & 1.1432 \\
\hline $\mathbf{T}(\square C)$ & $\mathbf{P}$ & $\mathbf{h}_{\mathrm{v}}$ & $\mathbf{h}_{1}$ & $\mathbf{S}_{1}$ & $\mathbf{S}_{\mathrm{v}}$ & $\mathrm{C}_{\mathrm{ps}}$ & $\mathrm{C}_{\mathrm{pv}}$ \\
\hline 263 & .19942 & 392.57 & 186.50 & 0.9499 & 1.7335 & 1.3152 & 0.8537 \\
\hline 313 & 1.0125 & 419.36 & 256.19 & 1.1898 & 1.7111 & 1.4975 & 1.1432 \\
\hline
\end{tabular}

\begin{tabular}{|l|l|l|l|l|l|l|l|}
\hline $\mathbf{T}(\square \mathbf{C})$ & $\mathbf{P}$ & $\mathbf{h}_{\mathbf{v}}$ & $\mathbf{h}_{\mathbf{l}}$ & $\mathbf{S}_{\mathbf{l}}$ & $\mathbf{S}_{\mathbf{v}}$ & $\mathbf{C}_{\mathbf{p s}}$ & $\mathbf{C}_{\mathbf{p v}}$ \\
\hline $\mathbf{2 7 3}$ & 0.29122 & 398.52 & 199.80 & 0.9927 & 1.7272 & 1.3406 & 0.8965 \\
\hline $\mathbf{3 1 3}$ & 1.0125 & 256.19 & 419.36 & 1.1898 & 1.7111 & 1.4975 & 1.1432 \\
\hline
\end{tabular}

\begin{tabular}{|l|l|l|l|l|l|l|l|}
\hline $\mathbf{T}(\square \mathbf{C})$ & $\mathbf{P}$ & $\mathbf{h}_{\mathbf{v}}$ & $\mathbf{h}_{\mathbf{l}}$ & $\mathbf{S}_{\mathbf{l}}$ & $\mathbf{S}_{\mathbf{v}}$ & $\mathbf{C}_{\mathbf{p s}}$ & $\mathbf{C}_{\mathbf{p v}}$ \\
\hline $\mathbf{2 8 3}$ & 0.41253 & 404.23 & 213.37 & 1.0478 & 1.7222 & 1.3699 & 0.94469 \\
\hline $\mathbf{3 1 3}$ & 1.0125 & 419.36 & 256.19 & 1.1898 & 1.7111 & 1.4975 & 1.1432 \\
\hline
\end{tabular}

MO9:

\begin{tabular}{|l|l|l|l|l|l|l|l|}
\hline $\mathbf{T}(\square \mathbf{C})$ & $\mathbf{P}$ & $\mathbf{h}_{\mathbf{v}}$ & $\mathbf{h}_{\mathbf{l}}$ & $\mathbf{S}_{\mathbf{l}}$ & $\mathbf{S}_{\mathbf{v}}$ & $\mathbf{C}_{\mathbf{p s}}$ & $\mathbf{C}_{\mathbf{p v}}$ \\
\hline $\mathbf{2 5 3}$ & 0.21361 & 414.08 & 171.55 & 0.89254 & 1.8837 & 1.3801 & 1.0347 \\
\hline $\mathbf{3 1 3}$ & 1.2818 & 433.43 & 260.84 & 1.2049 & 1.773 & 1.644 & 1.3678 \\
\hline
\end{tabular}

\begin{tabular}{|l|l|l|l|l|l|l|l|}
\hline $\mathbf{T}(\square \mathbf{C})$ & $\mathbf{P}$ & $\mathbf{h}_{\mathbf{v}}$ & $\mathbf{h}_{\mathbf{l}}$ & $\mathbf{S}_{\mathbf{l}}$ & $\mathbf{S}_{\mathbf{v}}$ & $\mathbf{C}_{\mathbf{p s}}$ & $\mathbf{C}_{\mathbf{p v}}$ \\
\hline $\mathbf{2 7 3}$ & 0.42215 & 420.87 & 199.78 & 0.99921 & 1.8354 & 1.4233 & 1.0818 \\
\hline $\mathbf{3 1 3}$ & 1.2818 & 433.43 & 260.84 & 1.2049 & 1.773 & 1.644 & 1.3678 \\
\hline $\mathbf{T}(\square \mathbf{C})$ & $\mathbf{P}$ & $\mathbf{h}_{\mathbf{v}}$ & $\mathbf{\mathbf { h } _ { 1 }}$ & $\mathbf{S}_{\mathbf{l}}$ & $\mathbf{S}_{\mathbf{v}}$ & $\mathbf{C}_{\mathbf{p s}}$ & $\mathbf{C}_{\mathbf{p v}}$ \\
\hline $\mathbf{2 6 3}$ & 0.30406 & 417.41 & 185.52 & 0.94637 & 1.8574 & 1.408 & 1.0643 \\
\hline $\mathbf{3 1 3}$ & 1.2818 & 433.43 & 260.84 & 1.2049 & 1.773 & 1.644 & 1.3678 \\
\hline
\end{tabular}

R410A

\begin{tabular}{|l|l|l|l|l|l|l|l|}
\hline $\mathbf{T}(\square \mathbf{C})$ & $\mathbf{P}$ & $\mathbf{h}_{\mathbf{v}}$ & $\mathbf{h}_{\mathbf{l}}$ & $\mathbf{S}_{\mathbf{l}}$ & $\mathbf{S}_{\mathbf{v}}$ & $\mathbf{C}_{\mathbf{p s}}$ & $\mathbf{C}_{\mathbf{p v}}$ \\
\hline $\mathbf{2 5 3}$ & 0.3984 & 419.43 & 170.22 & 0.88806 & 1.8709 & 1.438 & 0.99104 \\
\hline $\mathbf{3 1 3}$ & 2.4168 & 428.92 & 266.06 & 1.2201 & 1.7388 & 1.9357 & 1.8511 \\
\hline
\end{tabular}

\begin{tabular}{|l|l|l|l|l|l|l|l|}
\hline $\mathbf{T}(\square \mathbf{C})$ & $\mathbf{P}$ & $\mathbf{h}_{\mathbf{v}}$ & $\mathbf{h}_{\mathbf{l}}$ & $\mathbf{S}_{\mathbf{l}}$ & $\mathbf{S}_{\mathbf{v}}$ & $\mathbf{C}_{\mathbf{p s}}$ & $\mathbf{C}_{\mathbf{p v}}$ \\
\hline $\mathbf{2 6 3}$ & 0.5160 & 423.12 & 184.80 & 0.94403 & 1.848 & 1.4734 & 1.0615 \\
\hline $\mathbf{3 1 3}$ & 2.4168 & 428.92 & 266.06 & 1.2201 & 1.7388 & 1.9357 & 1.8511 \\
\hline
\end{tabular}

\begin{tabular}{|l|l|l|l|l|l|l|l|}
\hline $\mathbf{T}(\square \mathbf{C})$ & $\mathbf{P}$ & $\mathbf{h}_{\mathbf{v}}$ & $\mathbf{h}_{\mathbf{l}}$ & $\mathbf{S}_{\mathbf{l}}$ & $\mathbf{S}_{\mathbf{v}}$ & $\mathbf{C}_{\mathbf{p s}}$ & $\mathbf{C}_{\mathbf{p v}}$ \\
\hline $\mathbf{2 7 3}$ & 0.79668 & 426.25 & 199.77 & 0.99918 & 1.8267 & 1.57187 & 1.1442 \\
\hline $\mathbf{3 1 3}$ & 2.4168 & 428.92 & 266.06 & 1.2201 & 1.7388 & 1.9357 & 1.8511 \\
\hline $\mathbf{T}(\square \mathbf{C})$ & $\mathbf{P}$ & $\mathbf{h}_{\mathbf{v}}$ & $\mathbf{h}_{\mathbf{l}}$ & $\mathbf{S}_{\mathbf{l}}$ & $\mathbf{S}_{\mathbf{v}}$ & $\mathbf{C}_{\mathbf{p s}}$ & $\mathbf{C}_{\mathbf{p v}}$ \\
\hline $\mathbf{2 8 3}$ & 1.0835 & 428.67 & 215.23 & 1.0539 & 1.8061 & 1.5777 & 1.3439 \\
\hline $\mathbf{3 1 3}$ & 2.4168 & 428.92 & 266.06 & 1.2201 & 1.7388 & 1.9357 & 1.8511 \\
\hline
\end{tabular}

R407C:

\begin{tabular}{|l|l|l|l|l|l|l|l|}
\hline $\mathbf{T}(\square \mathbf{C})$ & $\mathbf{P}$ & $\mathbf{h}_{\mathbf{v}}$ & $\mathbf{h}_{\mathbf{l}}$ & $\mathbf{S}_{\mathbf{l}}$ & $\mathbf{S}_{\mathbf{v}}$ & $\mathbf{C}_{\mathbf{p s}}$ & $\mathbf{C}_{\mathbf{p v}}$ \\
\hline $\mathbf{2 5 3}$ & 0.27832 & 409.12 & 172.03 & 0.89453 & 1.8385 & 1.3577 & 0.89623 \\
\hline $\mathbf{3 1 3}$ & 1.7427 & 427.72 & 260.16 & 1.2018 & 1.7414 & 1.6554 & 1.3936 \\
\hline $\mathbf{T}(\square \mathbf{C})$ & $\mathbf{P}$ & $\mathbf{h}_{\mathbf{v}}$ & $\mathbf{h}_{\mathbf{l}}$ & $\mathbf{S}_{\mathbf{l}}$ & $\mathbf{S}_{\mathbf{v}}$ & $\mathbf{C}_{\mathbf{p s}}$ & $\mathbf{C}_{\mathbf{p v}}$ \\
\hline $\mathbf{2 6 3}$ & 0.40254 & 413.35 & 185.76 & 0.94739 & 1.819 & 1.3839 & 0.9483 \\
\hline $\mathbf{3 1 3}$ & 1.7427 & 427.72 & 260.16 & 1.2018 & 1.7414 & 1.6554 & 1.3936 \\
\hline
\end{tabular}




\begin{tabular}{|c|c|c|c|c|c|c|c|}
\hline$T(\square C)$ & $\mathbf{P}$ & $\mathbf{h}_{\mathrm{v}}$ & $\mathbf{h}_{1}$ & $S_{1}$ & $\mathbf{S}_{\mathrm{v}}$ & $\mathrm{C}_{\mathrm{ps}}$ & $C_{p v}$ \\
\hline 273 & 0.5651 & 417.28 & 199.79 & 0.99923 & 1.8017 & 1.4158 & 1.007 \\
\hline 313 & 1.7427 & 427.72 & 260.16 & 1.2018 & 1.7414 & 1.6554 & 1.3936 \\
\hline $\mathbf{T}(\square \mathbf{C})$ & $\mathbf{P}$ & $\mathbf{h}_{\mathrm{y}}$ & $\mathbf{h}_{\mathrm{H}}$ & $\mathbf{S}_{1}$ & $\mathbf{S}_{\mathrm{y}}$ & $\mathbf{C}_{n s}$ & $\mathbf{C}_{n \mathrm{nv}}$ \\
\hline 283 & 0.77291 & 427.72 & 214.15 & 1.0503 & 1.786 & 1.455 & 1.0794 \\
\hline 313 & 1.7427 & 427.72 & 260.16 & 1.2018 & 1.7414 & 1.6554 & 1.3936 \\
\hline
\end{tabular}

M50:

\begin{tabular}{|l|l|l|l|l|l|l|l|}
\hline $\mathbf{T}(\square \mathbf{C})$ & $\mathbf{P}$ & $\mathbf{h}_{\mathbf{v}}$ & $\mathbf{h}_{\mathbf{l}}$ & $\mathbf{S}_{\mathbf{l}}$ & $\mathbf{S}_{\mathbf{v}}$ & $\mathbf{C}_{\mathbf{p s}}$ & $\mathbf{C}_{\mathbf{p v}}$ \\
\hline $\mathbf{2 5 3}$ & 0.22832 & 422.81 & 163.23 & 0.86133 & 1.8756 & 1.7757 & 1.1161 \\
\hline $\mathbf{3 1 3}$ & 1.3362 & 465.80 & 279.48 & 1.267 & 1.8565 & 2.1591 & 1.6124 \\
\hline
\end{tabular}

\begin{tabular}{|l|l|l|l|l|l|l|l|}
\hline $\mathbf{T}(\square \mathbf{C})$ & $\mathbf{P}$ & $\mathbf{h}_{\mathbf{v}}$ & $\mathbf{h}_{\mathbf{l}}$ & $\mathbf{S}_{\mathbf{l}}$ & $\mathbf{S}_{\mathbf{v}}$ & $\mathbf{C}_{\mathbf{p s}}$ & $\mathbf{C}_{\mathbf{p v}}$ \\
\hline $\mathbf{2 6 3}$ & 0.32587 & 429.63 & 181.25 & 0.93067 & 1.87672 & 1.8186 & 1.1705 \\
\hline $\mathbf{3 1 3}$ & 1.3362 & 465.80 & 279.48 & 1.267 & 1.8565 & 2.1591 & 1.6124 \\
\hline
\end{tabular}

\begin{tabular}{|l|l|l|l|l|l|l|l|}
\hline $\mathbf{T}(\square \mathbf{C})$ & $\mathbf{P}$ & $\mathbf{h}_{\mathbf{v}}$ & $\mathbf{h}_{\mathbf{l}}$ & $\mathbf{S}_{\mathbf{l}}$ & $\mathbf{S}_{\mathbf{v}}$ & $\mathbf{C}_{\mathbf{p s}}$ & $\mathbf{C}_{\mathbf{p v}}$ \\
\hline $\mathbf{2 7 3}$ & 0.45191 & 437.21 & 199.72 & 0.99898 & 1.8614 & 1.8669 & 1.2321 \\
\hline $\mathbf{3 1 3}$ & 1.3362 & 465.80 & 279.48 & 1.267 & 1.8565 & 2.1591 & 1.6124 \\
\hline
\end{tabular}

\begin{tabular}{|l|l|l|l|l|l|l|l|}
\hline $\mathbf{T}(\square \mathbf{C})$ & $\mathbf{P}$ & $\mathbf{h}_{\mathbf{v}}$ & $\mathbf{h}_{\mathbf{l}}$ & $\mathbf{S}_{\mathbf{l}}$ & $\mathbf{S}_{\mathbf{v}}$ & $\mathbf{C}_{\mathbf{p s}}$ & $\mathbf{C}_{\mathbf{p v}}$ \\
\hline $\mathbf{2 8 3}$ & 0.6111 & 444.69 & 218.70 & 1.0665 & 1.8583 & 1.9221 & 1.3028 \\
\hline $\mathbf{3 1 3}$ & 1.3362 & 465.80 & 279.48 & 1.267 & 1.8565 & 2.1591 & 1.6124 \\
\hline
\end{tabular}

IV. Result

COP OF DIFFERENT REFRIGERANTS AT DIFFERENT EVAPORATOR TEMPERATURES

\begin{tabular}{|c|c|c|c|c|c|c|c|}
\hline TEMP(K) & R22 & R134a & M09 & R410A & R407C & R290 & M50 \\
\hline 253 & 3.15 & 3.063 & 2.766 & 2.93 & 2.975 & 3.02 & 2.86 \\
\hline 258 & 3.59 & 3.501 & 3.149 & 3.33 & 3.39 & 3.44 & 3.2856 \\
\hline 263 & 4.11 & 4.027 & 3.619 & 3.83 & 3.94 & 3.96 & 3.79 \\
\hline 268 & 4.75 & 4.679 & 4.19 & 4.43 & 4.50 & 4.603 & 4.429 \\
\hline 273 & 5.042 & 5.49 & 4.917 & 5.19 & 5.28 & 5.40 & 5.21 \\
\hline 278 & 6.59 & 6.55 & 5.84 & 6.18 & 6.28 & 6.42 & 6.248 \\
\hline 283 & 7.98 & 7.95 & 7.09 & 7.15 & 7.62 & 7.80 & 7.6225 \\
\hline
\end{tabular}

From the above table it is observed that R407c and R410a is very much close to R22 in terms of COP. For lower evaporator temperature COP of R410a is $6 \%-7 \%$ lower, and at higher temperature of evaporator it is only $4 \%$ lower than R22.

Similar is the case with R407c at lower evaporator temperature the COP is 5\%-6\%lower than R22.

M09 COP is 9\%-10\% less than R134a at given evaporator temperature range. M50 has COP 6\%-7\% lower than that of R134a at lower evaporator temperature and at higher evaporator temperature it is $4 \%-5 \%$ lower. Cop of propane is $1.6 \%-1.9 \%$ lower than that of R134a in the given range of evaporator temperature.

REFRIGERATION EFFECT OF VARIOUS REFRIGERANTS AT DIFFERENT EVAPORATOR TEMPERATURE 


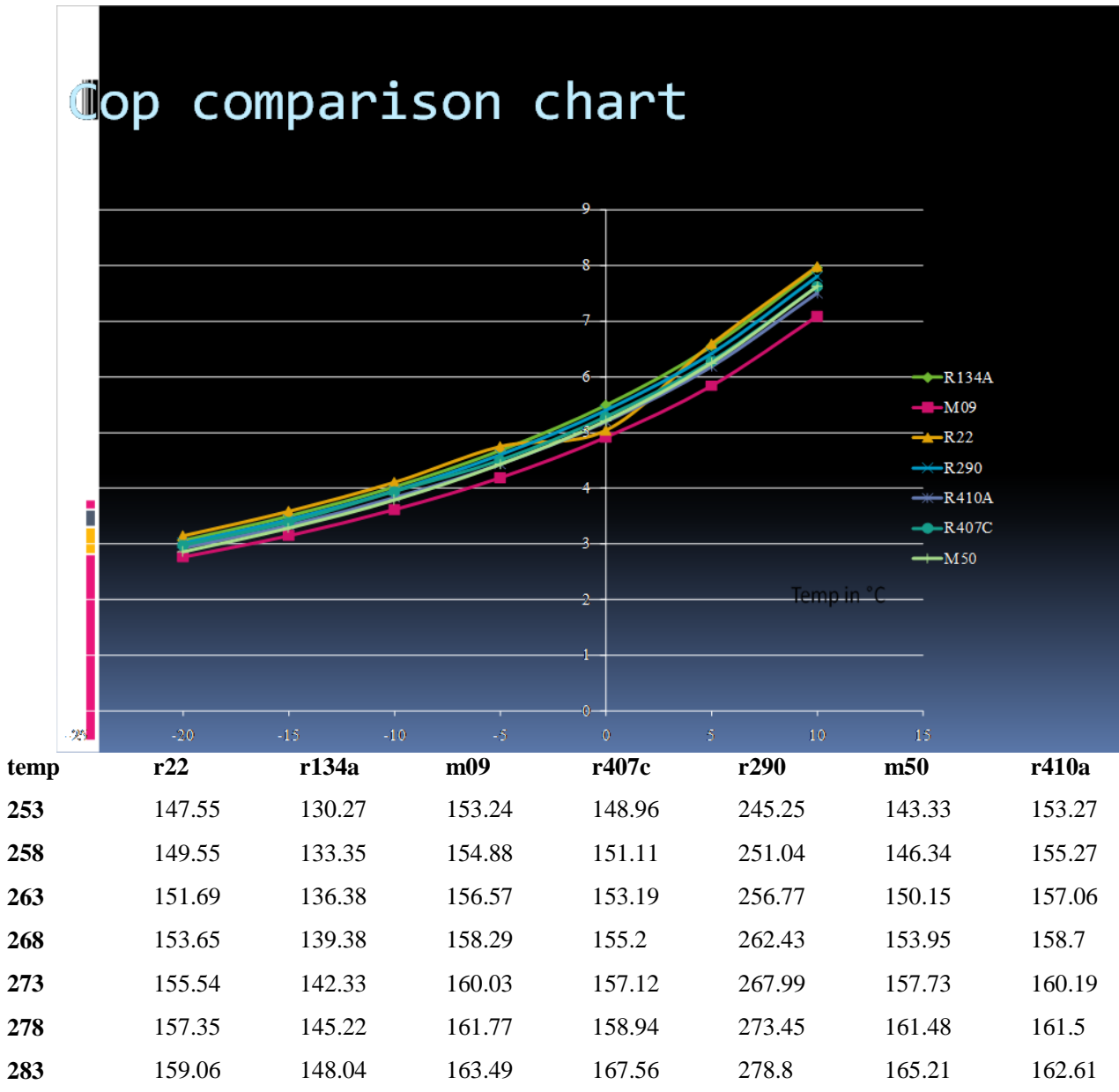

Taking mass flow rate of refrigerant $\mathrm{m}=1 \mathrm{~kg} / \mathrm{min}$

Unit of $\mathrm{RE}$ is $\mathrm{KJ} / \mathrm{min}$

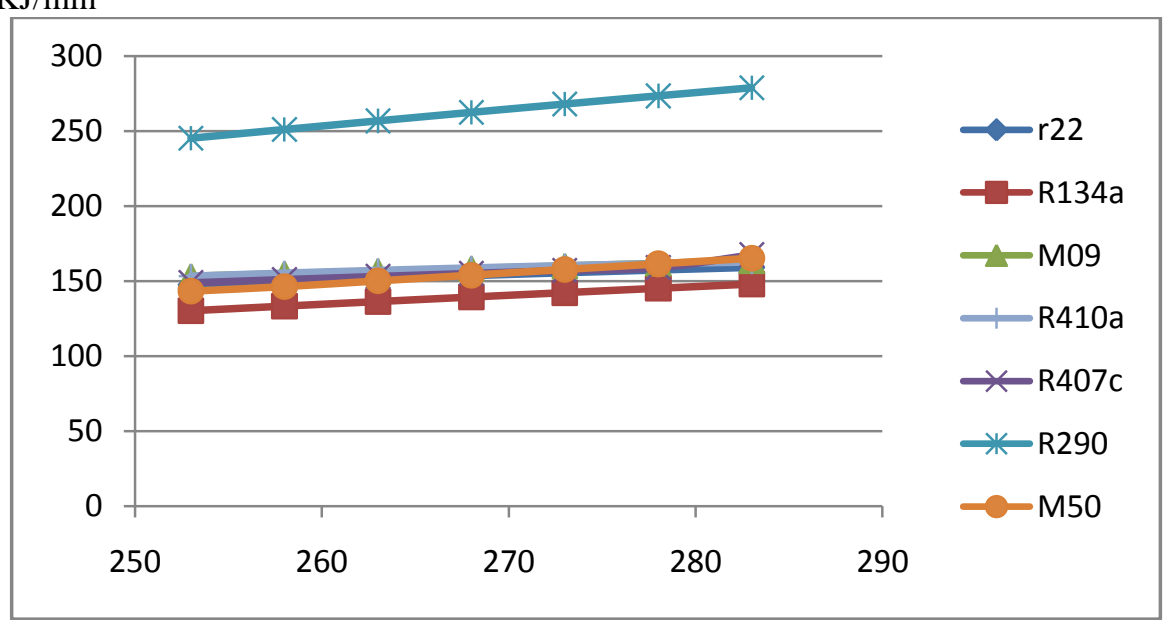

From the above table of refrigeration effect it is observed that refrigeration effect of R407c is $0.95 \%-1.043 \%$ greater than that of R22 in the temperature range of $253 \mathrm{~K}$ to $278 \mathrm{~K}$. Refrigeration effect of R410a is $3.28 \%$ $3.87 \%$ higher than that of R22 at the lower evaporator temperature, whereas at higher evaporator temperature it is $2.6 \%$ to $2.9 \%$ higher.

Refrigeration effect of M09 and R290 is also greater than that of R134a.For M09 it is 10\% to 18\% higher in the given range of evaporator temperature. For R290 refrigeration effect values is remarkably high. It is $88 \%$ to 88.4\% higher than R143a in the given range of temperature. Refrigeration effect of R290 is the greatest of all the refrigeration under analysis. M50 has higher refrigeration effect values in the give temperature range. It is $10 \%$ to $12 \%$ greater than that of R134a. 


\section{COMPRESSOR WORK OF VARIOUS REFRIGERANTS AT DIFFERENT EVAPORATOR TEMPERATURE}

Taking mass flow rate $\mathrm{m}=1 \mathrm{~kg} / \mathrm{min}$

Unit of compressor work is $\mathrm{kW}$

\begin{tabular}{|l|l|l|l|l|l|l|l|}
\hline TEMP(K) & R22 & R134a & M09 & R410a & R407C & R290 & M50 \\
\hline $\mathbf{2 5 3}$ & 0.778 & 0.742 & 0.924 & 0.872 & 0.834 & 1.353 \\
\hline $\mathbf{2 5 8}$ & 0.694 & 0.635 & 0.819 & 0.775 & 0.743 & 0.817 \\
\hline $\mathbf{2 6 3}$ & 0.614 & 0.564 & 0.721 & 0.683 & 0.655 & 1.213 & 1.079 \\
\hline $\mathbf{2 6 8}$ & 0.539 & 0.496 & 0.574 & 0.596 & 0.574 & 0.742 \\
\hline $\mathbf{2 7 3}$ & 0.508 & 0.432 & 0.495 & 0.514 & 0.495 & 0.950 & 0.827 \\
\hline $\mathbf{2 7 8}$ & 0.436 & 0.369 & 0.461 & 0.435 & 0.421 & 0.579 \\
\hline $\mathbf{2 8 3}$ & 0.369 & 0.310 & 0.384 & 0.361 & 0.236 & 0.709 & 0.595 \\
\hline
\end{tabular}

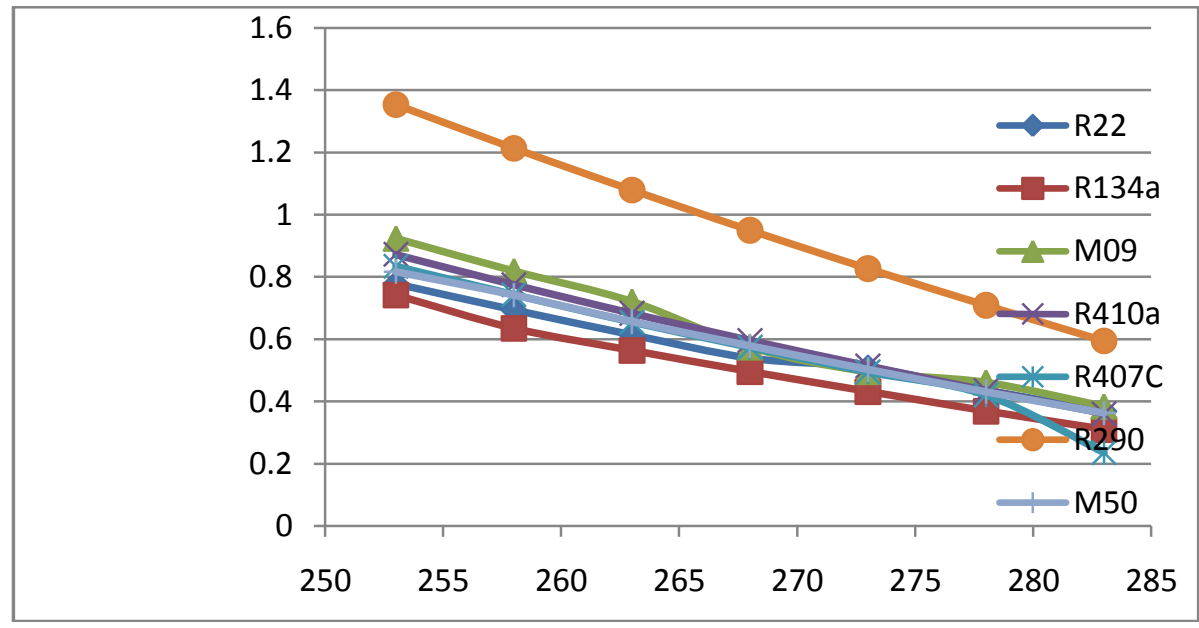

The above table shows the compressor work of refrigerants under analysis and following observations are made. In the temperature range of $258 \mathrm{~K}$ to $273 \mathrm{~K}$ compressor work for $\mathrm{R} 410 \mathrm{a}$ is higher than R22 by $10 \%$ to $12 \%$. But in the temperature range of $278 \mathrm{~K}$ to $283 \mathrm{~K}$ compressor work is lesser by $1 \%$ to $3 \%$.

For R407c compressor work is higher than that of R22 in the temperature range of $253 \mathrm{~K}$ to $268 \mathrm{~K}$ but it becomes less than that of R22 in the higher evaporator temperature range.

When compared with R134a, M09 has higher compressor work. It is $20 \%$ to $25 \%$ greater in the given temperature range.

R290 has compressor work values much higher than that of R143a at all range of evaporator temperature.

\section{Conclusion}

It is observed from the above calculations that R410a and R407c can be the good replacements for R22. COP values of both the refrigerant are comparable to R22. Besides refrigeration effect is greater and compressor work of these two refrigerants is less than that of R22 in the higher range of evaporator temperature.

M09 and R290 seem to be the potential substitutes for R134a. It can be seen from the results that COP of R290 is very much same as at of R134a in the given range of evaporator temperature. And values refrigeration effect is more than that of R134a.

R290 (propane) is a hydrocarbon. Hydrocarbons are very flammable. If used in very large amount it can be a dangerous refrigerant. Hence R290 should not be used in industrial purpose when a large amount of refrigerant needs to be used although it is beneficial when used for household purpose or small units.

M09 has very less percentage of hydrocarbons. $91 \%$ of it consists of R134a whereas rest of the 9\% consists of the mixture of propane and isobutene. Because of very less amount of hydrocarbons used, it can be used in lagre applications. Besides it refrigerant is more economic when its cost is considered. It can also be used for retrofitting. Hence M09 could be a potential substitute for R134a.

\section{Biblography}

[1]. Improved energy efficiency for CFC domestic refrigerators retrofitted with ozone friendly HFC/HC refrigerant mixture by S. Joseph Sekhar, D. Mohan Lal, and S. Renganarayanan.

[2]. Comparative performance study of vapour compression refrigeration system with R22/R134a/R410a/R407c/M20 by Vaibhavjain, S.S. Kachhwaha, R.S. Mishra.

[3]. The use of hydrocarbons propane and isobutane in refrigeration system by Jacqueline BianconCopetti, Mario Henrique Macagnan.

[4]. Refrigerants environmental propertieshttp://www.engineeringtoolbox.com/refrigerants-properties-d_145.html

[5]. Global warming potential. http://en.wikipedia.org/wiki/Global-warming_potential

[6]. Global change: ozone depletion potential_ss.snre.umich.edu/css doc/GlobalChange OD.ppt

[7]. Ozone depletion potentialhttp://en.wikipedia.org/wiki/Ozone_depletion_potential 
[8]. Refprop(NIST) help guide

[9]. Vapour compression refrigeration cycle- RK RAJPUT

[10]. Vapour compression refrigeration cycle: understanding refrigerationhttp://www.alephzero.co.uk/ref/vapcomcyc.htm

[11]. Montreal protocol: the montreal protocol on substances that deplete layer.http://www.epa.gov/ozone/intpol/http://en.wikipedia.org/wiki/Montreal Protocol

[12]. Experimental analysis of a new refrigerant mixture as drop-in replacement for CFC12 and HFC134a by K. Mani, V. Sel 